\title{
Time-Resolved Studies of Excitonic Dynamics in a Wide II-VI Quantum Well by a Femtosecond Pump-Probe Reflectivity
}

\author{
A. Trajnerowicz ${ }^{a}$, A. Golnik ${ }^{a}$, P. Kossacki ${ }^{a}$, W. Pacuski ${ }^{a}$ \\ AND T. WOJTOWICZ ${ }^{b}$ \\ ${ }^{a}$ Institute of Experimental Physics, Warsaw University \\ Hoża 69, 00-681 Warsaw, Poland \\ ${ }^{b}$ Institute of Physics, Polish Academy of Sciences \\ al. Lotników 32/46, 02-668 Warsaw, Poland
}

We present time-dependent reflectance spectra of wide $(\mathrm{Cd}, \mathrm{Mn}) \mathrm{Te} /(\mathrm{Cd}, \mathrm{Mg}) \mathrm{Te}$ quantum wells. Interactions between excitons, trions, and carriers are studied for exciton densities up to $10^{11} \mathrm{~cm}^{-2}$. The resonant excitation at different excitonic lines is analyzed.

PACS numbers: 78.67.De, 71.35.Pq, 78.47.+p

\section{Introduction}

The pump-probe technique is a valuable tool to obtain detailed information on excitation and photoluminescence of quantum wells (QW) containing carrier gas. It is particularly useful in a study of many body effects. The use of ultrafast excitation allows combining extremely high excitations with limited thermal effects. In the previous works regarding CdTe and $(\mathrm{Cd}, \mathrm{Mn}) \mathrm{Te} \mathrm{QW}[1,2]$ the effects of phase space filling, oscillator strength stealing, and spin dependent screening were discussed.

In the present work we compare the wells with electron and hole gas. The use of a wider QW also allowed us to study the influence of the strong excitation on the excitonic states composed of carriers from higher subbands. 


\section{Samples and experiment}

We will compare results obtained for two samples, which were grown by molecular beam epitaxy (MBE) and containing single quantum wells of $\mathrm{Cd}_{1-x} \mathrm{Mn}_{x}$ Te embedded between $(\mathrm{Cd}, \mathrm{Mg}) \mathrm{Te}$ barriers. The first sample has a $80 \AA$ wide QW with $0.21 \%$ of $\mathrm{Mn}$ and iodine $\delta$-doping ensuring the presence of electron gas in QW. The sample was well characterized in Ref. [3]. The second sample contains a $160 \AA$ wide QW with $0.72 \% \mathrm{Mn}$ and has no intentional doping. QW is $p$-type because of the surface states on $500 \AA$ cap [4].

The samples were mounted stress-free in a cryostat with a superconducting magnet. The measurements were taken in a temperature of about $1.6 \mathrm{~K}$.

The pump-probe reflectivity experiment was performed in the configuration schematically shown in Fig. 1. $\mathrm{A} \mathrm{Ti}^{3+}: \mathrm{Al}_{2} \mathrm{O}_{3} 60$ fs pulse laser with a repetition

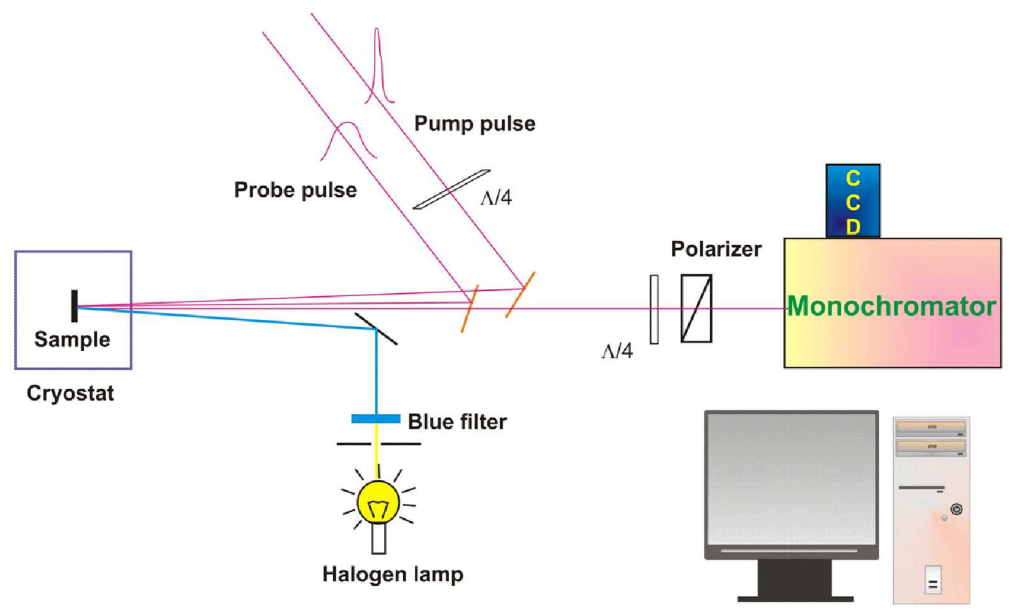

Fig. 1. The scheme of the setup of pump-probe reflectivity.

rate of $76 \mathrm{MHz}$ was used as a light source for both pump and probe beams. Probe one remained spectrally broad (FWHM of about $40 \mathrm{~nm}$ ) and pump beam was spectrally narrowed on a dispersive set with a diffraction grating and adjustable slit. The slit allowed the choice of photon energy and FWHM of the pump pulse. The probe pulse was sent through a delaying line, which allowed us to control precisely the delay between the pump and the probe pulse. The power of each beam was controlled separately.

The pump beam was circularly polarized; while the probe beam remained linearly polarized and it was detected after the sample at both circular polarizations. The pump-probe experiment was done in zero magnetic field, so only a mutual orientation of circular polarizations of the pump and the reflected probe pulses is important, therefore we will state only whether the pulses were co-polarized or cross-polarized. 
The reflected probe pulse was detected by a CCD camera attached to a monochromator.

The additional blue light illumination of the samples (tungsten halogen lamp with blue filter) allowed the control of carrier gas concentration in the QW [5].

\section{Results}

Figure 2 presents spectra of both samples: (a) $80 \AA \mathrm{QW}$, (b) $160 \AA \mathrm{QW}$ in negative delay (the probe pulse comes earlier than the pump pulse). In Fig. 2a. we can identify three states: the lowest energetic negatively charged exciton, strong heavy hole exciton, and weak light hole exciton. In Fig. $2 b$ there are: positively charged exciton, strong neutral heavy hole exciton, light hole exciton, and two weak heavy hole excitons.

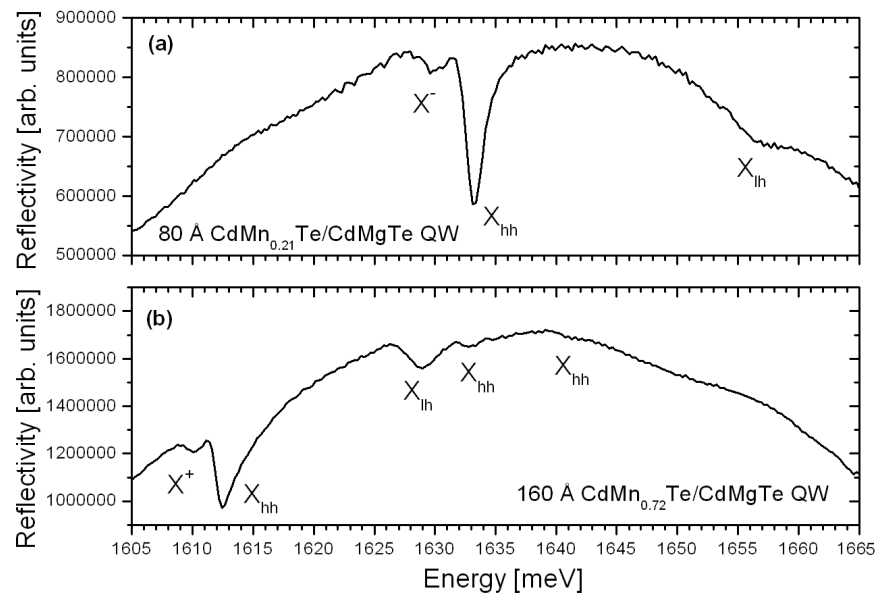

Fig. 2. Reflectivity spectra of the probe pulse of (a) $80 \AA \mathrm{CdMnTe} / \mathrm{CdMgTe} \mathrm{QW}$ and (b) $160 \AA \mathrm{CdMnTe} / \mathrm{CdMgTe} \mathrm{QW}$.

According to small differences in spectra for some resonances and polarization configurations it is better to analyze difference spectra which come from subtracting the spectrum obtained at negative delay from each one. In our analysis we subtracted a first spectrum taken at -33 ps. Further we focused on the behavior of the absorption near the zero delay time, when the densities of photo-generated carrier are maximum.

\section{1. n-type $80 \AA$ quantum well}

Pumping resonantly charged exciton states we observed similar changes in behavior of lines as in previous works [1]. In co-polarization (co-polarized beams of the pump and the probe) a decrease of $X$-state is seen, which is caused by a saturation of the transition (saturation of free electrons). Simultaneously, the absorption of neutral heavy-hole (hh) exciton increases. This was interpreted as 
a result of a weakening of screening by free carriers bounded now into trions. In cross-polarization the trion line slightly increases - the weakening of screening, hh exciton line initially does not change - it suggests that screening of exciton is more efficient by electrons with an opposite spin than those creating excitons [1].

In the case of excitation of neutral hh exciton state (Fig. 3) there is a decrease in intensity of the trion transition in both polarization configurations. The hh exciton line increases in co-polarization - the weakening of screening by carriers being bounded into trions. In cross-polarization this transition decreases biexcitons are being created [1].

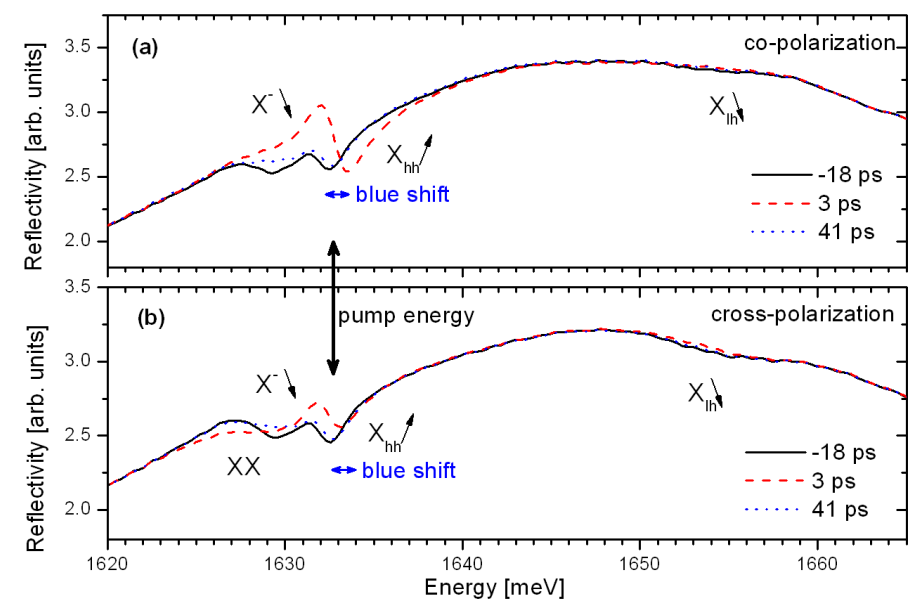

Fig. 3. Reflectivity spectra of $80 \AA \mathrm{CdMnTe} / \mathrm{CdMgTe} \mathrm{QW}$ in three different delays between pump and probe pulses in (a) co-polarization configuration and (b) cross-polarization configuration.

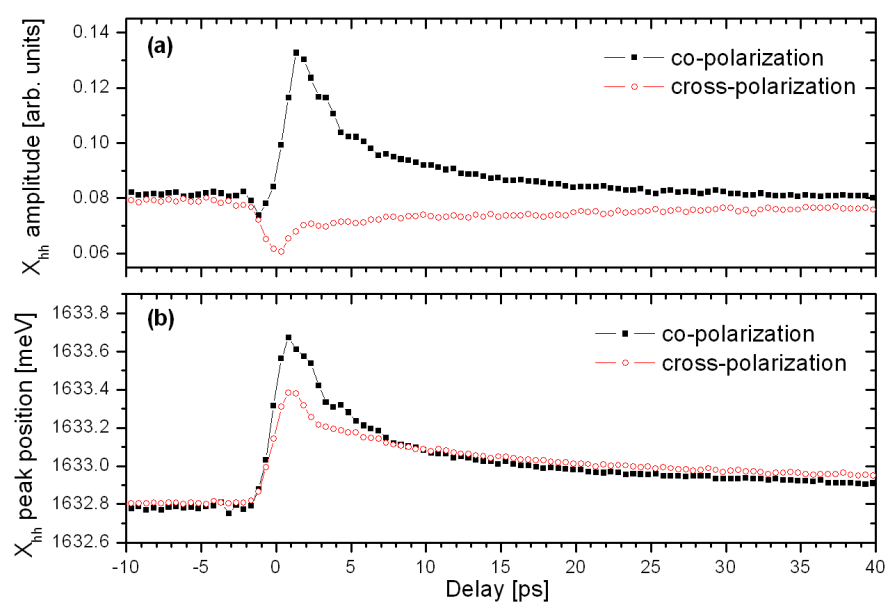

Fig. 4. Amplitude (a) and energy (b) of the hh exciton line of $80 \AA$ CdMnTe/CdMgTe QW for different delays between pump and probe pulses (pump at $X_{\mathrm{hh}}$ ). 
For both experiments (resonant excitation of trions and hh excitons) the intensity of the light-hole (lh) exciton weakly decreases. When lh exciton state is resonantly excited there is a decrease in intensity of all transitions in both configurations. Additionally blue shifts of hh exciton line are observed (Fig. 3 and Fig. 4b). The blue shift in cross-polarization is probably caused by phase space filling for the electrons. The pump pulse creates lh excitons and the probe one creates some hh excitons with electrons of the same spin.

\section{2. p-type 160 A quantum well}

In the case of the second sample five different excitonic lines were seen clearly enough to be analyzed (Fig. 2b). The behavior of all lines is studied in dependence of resonant excitation of each state. When the pump pulse is in a resonance with charged exciton or neutral hh exciton similar changes are observed as in the previous sample and earlier studies $[1,2]$. However the excitation in higher energetic hh excitons did not affect the other lines significantly. Only a decrease in intensity of all other lines, the same in both polarizations, was observed.

An interesting effect was found for light hole exciton line. When the fundamental state of hh exciton was pumped, the lh $X$ line exhibited both change of the intensity and shift. The most striking blue shift is seen in cross-polarization (Fig. 5b and Fig. 6b). It is as high as $0.4 \mathrm{meV}$, while the shift in co-polarization is below $0.2 \mathrm{meV}$. We interpret this as a sign of the phase space filling for electrons. The pump creates hh excitons which consist of ( $\pm 1 / 2$ electrons and $\pm 3 / 2$ holes). The cross-polarized probe involves lh excitons with the same $\pm 1 / 2$ electron and $\pm 1 / 2$ hole. Similar difference in blue shifts is observed for hh exciton lines, but then the effect in co-polarizations is dominant. In co-polarization the blue shift is

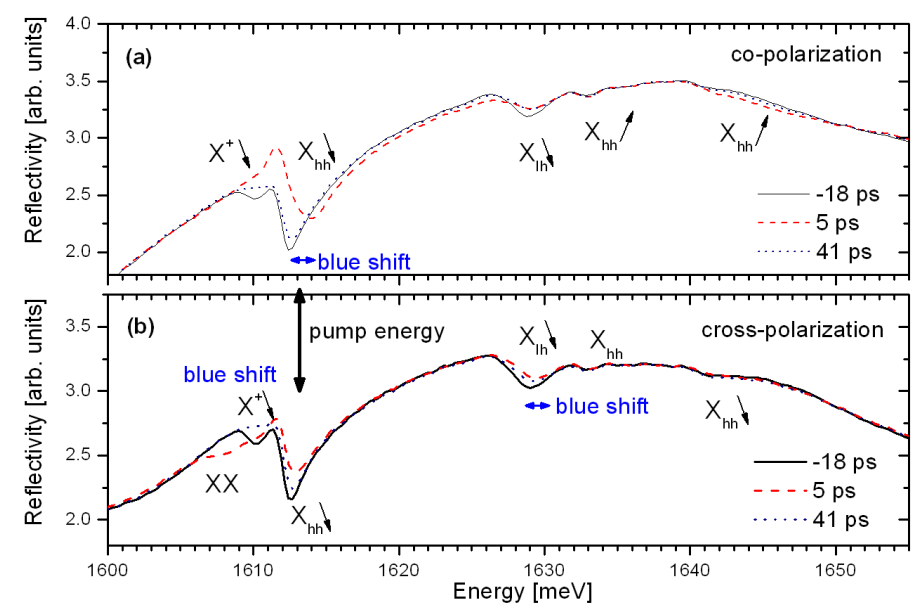

Fig. 5. Reflectivity spectra of $160 \AA$ CdMnTe/CdMgTe QW in three different delays between pump and probe pulses in (a) co-polarization configuration and (b) cross-polarization configuration. 


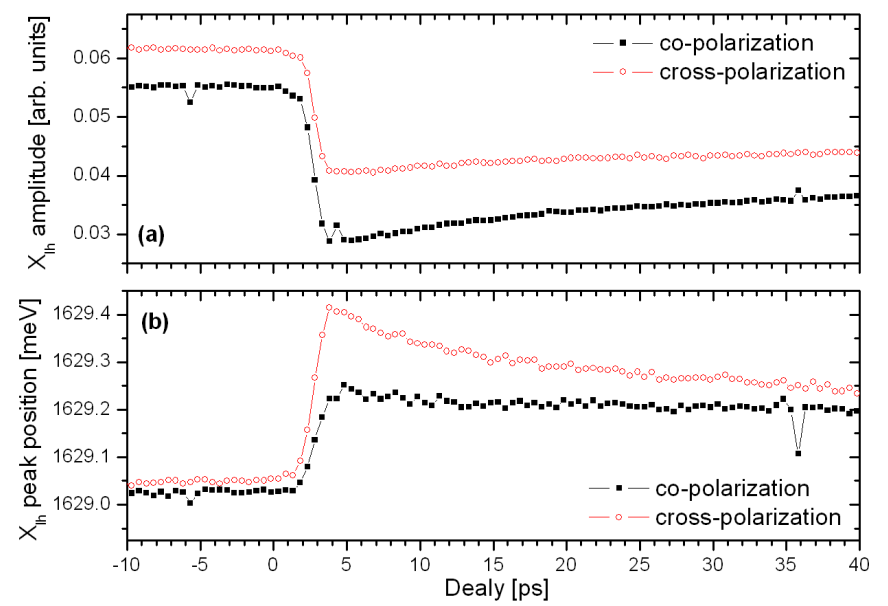

Fig. 6. Amplitude (a) and energy (b) of the lh exciton line of $160 \AA$ CdMnTe/CdMgTe QW for different delays between pump and probe pulses (pump at $X_{\mathrm{lh}}$ ).

clearly seen, while in cross-polarization it is not (Fig. 5). For the $n$-type $80 \AA \mathrm{QW}$ the blue shifts are in both configurations (Fig. 3 and Fig. 4b). This could be an effect of the type of the sample or the well width. We treat this as an argument for the leading role of the electron phase space filling in the blue shifts observed in absorption.

\section{Conclusion}

We studied the $n$-type $80 \AA$ and $p$-type $160 \AA$ (Cd,Mn)Te/(Cd,Mg)Te quantum wells in the condition of strong optical excitation. The influence of high population of the photo-created carriers was investigated for different hh and $\mathrm{lh}$ excitonic transitions. Pumping the hh exciton in one polarization causes the blue shift of lh exciton line in second polarization; the same happens when the lh exciton transition is excited.

The difference in the blue shifts of hh exciton lines for two polarization configurations is observed for $p$-type $160 \AA$ wide $\mathrm{QW}$, while for $n$-type $80 \AA \mathrm{QW}$ there is no such difference. To prove whether it is an effect of the width or the type, further experiments are needed.

\section{Acknowledgment}

This work has been supported by the State Committee for Scientific Research grant 2P03B 00225. 


\section{References}

[1] P. Płochocka, P. Kossacki, W. Maślana, J. Cibert, S. Tatarenko, C. Radzewicz, J.A. Gaj, Phys. Rev. Lett. 92, 177402 (2004); P.P. Kossacki, P. Płochocka, B. Piechal, W. Maślana, A. Golnik, J. Cibert, S. Tatarenko, J.A. Gaj, Phys. Rev. B 72, 035340 (2005).

[2] M.T. Portella-Oberli, V. Ciulin, J.H. Berney, B. Deveaud, M. Kutrowski, T. Wojtowicz, Phys. Rev. B 69, 235311 (2004).

[3] M. Kutrowski, T. Wojtowicz, P. Kossacki, V. Ciulin, J. Kossut, Phys. Status Solidi B 229, 791 (2002).

[4] W. Maslana, P. Kossacki, M. Bertolini, H. Boukari, D. Ferrand, S. Tatarenko, J. Cibert, J.A. Gaj, Appl. Phys. Lett. 82, 1875 (2003).

[5] P. Kossacki, H. Boukari, M. Bertolini, D. Ferrand, J. Cibert, S. Tatarenko, J.A. Gaj, B. Deveaud, V. Ciulin, M. Potemski, Phys. Rev B 70, 195337 (2004). 